\title{
New Algorithms for Optimizing and Linking Conical
}

\section{Intersection Points}

Fabrizio Sicilia ${ }^{\S}$,Lluís Blancafort ${ }^{\dagger,}$, Michael J. Bearpark ${ }^{\S}$, and Michael A. Robb ${ }^{\S, *}$

Department of Chemistry, Imperial College, London SW7 2AZ, United Kingdom, Institut de Quimica Computational and Departament de Quimica, Universitat de Girona, E-17071 Girona, Spain

Corresponding author: mike.robb@imperial.ac.uk, 1luis.blancafort@udg.edu

RECEIVED DATE 
Abstract: In this paper we present two new algorithms to study the extended nature of the crossing seam. The first algorithm is designed to optimize conical intersection geometries: both minima and saddle points along the crossing seam. In addition, this method can be employed to carry out conical intersection optimization with geometrical constraints. We demonstrate the potentialities of such algorithm on different crossing seams of benzene, z-penta-3,5-diemminium and 1,3-butadiene. The second algorithm has instead been designed to explicitly compute the intersection-space minimum energy coordinate. Our computations show how an intersection seam and the energy along it can unambiguously defined. A finite region of the $S_{0} / S_{1} 1,3$-butadiene crossing seam has been mapped out, showing the connectivity amongst three conical intersection structures, of which two never reported previously. 


\section{Introduction}

In the past two decades conical intersection geometries have been proven to play a central role in our understanding of photochemical reactions (see for instance Refs. ${ }^{1-4}$ ). These structures take part in phochemical processes in a similar manner as the transition state geometries are involved in a thermochemical reaction ${ }^{2}$. Up to date, numerous conical intersection structures have been located and shown to be involved in several non-radiative processes (see for instance Refs. ${ }^{1-5}$ ).

Conical intersections are not isolated points, but rather are connected along an (n-2)dimensional hyperline, where $n$ is the number of internal degrees of freedom. Recent studies have showed that decay can also occur at a higher energy point along the crossing hyperline (see for example Refs. ${ }^{6,7}$ ). Thus, an accurate investigation of intersection space ${ }^{8}$, which is the space where the two electronic states are degenerate, becomes crucial.

In this spirit, we recently developed a new methodology to compute the curvature of the crossing seam energy, such that saddle points could be distinguished from minima point within the intersection space ${ }^{9-11}$. In addition, from these frequency calculations we could compute the motions corresponding to the imaginary frequencies. These intersection-space vibrational modes ${ }^{10}$ were then used to suggest connections amongst several conical intersection points belonging to the same intersection space. In this paper, we describe two complementary tools to study the intersection space. The first algorithm is designed to optimize conical intersection structures using realistic second derivatives 
and updating. The second method is instead capable to compute the minimum energy path connecting three conical intersection structures, e.g. two minima and a saddle point along the crossing seam. Thus, with the algorithms described in this paper we present a complete series of tools capable of investigating the energy within the intersection space in the same way that one would study a single Born-Oppenheimer potential energy surface (see for instance ${ }^{12}$ ).

Over the years, many different approaches have been proposed to optimize structures where two electronic states become degenerate. The algorithms currently available are based upon either Lagrange-Newton methods (see for example Refs. ${ }^{13,14}$ ) or projection methods (see for instance Refs. ${ }^{15,16}$ ). The algorithms belonging to the first class have the common feature of using variations of the classical Lagrangian multipliers method ${ }^{17,18}$. In contrast, the projection methods are designed to reach the energy degeneracy, by means of a displacement within the branching space ${ }^{8}$, and to optimize simultaneously the energy of the excited state within the intersection space ${ }^{8}$.

A typical algorithm, based on the projection matrices, uses a gradient composed of two distinct parts ${ }^{15}$. The first part consists of the normalized gradient difference vector, which is one of the two first-order degeneracy lifting directions ${ }^{1}$, weighted by twice the energy gap. This term is responsible of minimizing the energy difference between the two crossing states. The second part of the gradient optimizes the excited state energy within the intersection space. Thus a critical point on the intersection hyper-line can be located. 
The use of projection matrix ensures the orthogonality between the two parts of the composite gradient. However, such orthogonality may be lost in computing a quasiNewton-Raphson displacement, as consequence of an ill-conditioned approximate Hessian. In other words, the displacement computed may have components in both branching and intersection space. This problem becomes troublesome in the region where the two electronic states become almost degenerate. Here, the displacement components within the branching plane will lift the degeneracy. The algorithm described in this paper therefore uses a combination of displacements taken within the two orthogonal spaces rather than two gradients. Although this idea is related to the theoretical development originally proposed by Anglada et al. ${ }^{19}$ and recently reviewed and extended by De Vico et al. ${ }^{20}$, the actual implementation of the proposed algorithm is substantially different, as we will discuss in the next section.

In the present algorithm, as the energy difference drops below a set threshold, a Newton-Raphson displacement, taken within the intersection space, is combined with a step along the gradient difference vector. When the energy is above the given threshold, a standard projection method ${ }^{15}$ is used. As we will discuss in Section 4, the proposed algorithm shows a faster and smoother overall convergence to the minimum conical intersection geometry when compared with two previously reported algorithms ${ }^{15,19}$. In addition, our implementation enables one to optimize conical intersection geometries along a given constrained redundant coordinate ${ }^{21-23}$. Finally, this algorithm and the possibility of computing analytically an intersection-space Hessian ${ }^{10}$ can be combined with the transition state search algorithm (for a recent review see Ref. ${ }^{12}$ ) implemented in 
Gaussian package ${ }^{24}$. To summarize, the algorithm proposed in this paper is capable of locating both minima and saddle points within the intersection space, as well as of optimizing conical intersection points along a given constrained geometrical variable.

Using two independent displacements, one within the intersection space and one along the gradient difference vector, we have also designed a method to compute a coordinate analogous to the intrinsic reaction coordinate ${ }^{25-27}$, but confined to the intersection space. Although such coordinate may be not physically meaningful (see for example ${ }^{28}$ and following comments), it represents a unique way to define the intersection space. In Section 4 , we will present the study carried out to link a new saddle point found on the $\mathrm{S}_{0} / \mathrm{S}_{1}$ crossing seam of 1,3-butadiene with two low lying conical intersections. 


\section{Theory}

In this section we will present two different algorithms. The first method is designed to improve the existent conical intersection optimization algorithms. The second allows the computation of an intersection space coordinate analogous to the intrinsic reaction coordinate ${ }^{25-27}$. In what follows, we will assume that the two crossing states intersect along a (n-2) hyperline, where $n$ is the number of internal coordinates. In other words, the two electronic states cross each other at all the nuclear configurations belonging to an ( $n$ 2)-dimensional intersection space, where $n$ is the number of internal coordinates of the molecular system ${ }^{29}$.

\subsection{Optimization of stationary points along a crossing seam.}

At a conical intersection point, a displacement along two directions is capable of lifting the degeneracy at first order: the gradient difference [Eq.(1a)] and the interstate coupling [Eq.(1b)] (see for example Refs. ${ }^{1,4,13,30,31}$ ).

$$
\begin{aligned}
& \mathbf{x}_{1}=\frac{\partial\left(E_{2}-E_{1}\right)}{\partial \mathbf{q}} \\
& \mathbf{x}_{2}=\left\langle\mathbf{C}_{1}\left|\frac{\partial \mathbf{H}}{\partial \mathbf{q}}\right| \mathbf{C}_{2}\right\rangle
\end{aligned}
$$

In Eq.(1), $\mathbf{C}_{\mathbf{1}}$ and $\mathbf{C}_{\mathbf{2}}$ are the CI eigenvectors in the MC-SCF problem. The vectors $\mathbf{x}_{\mathbf{1}}$ and $\mathbf{x}_{2}$ span the branching plane ${ }^{8}$, also referred to as the $g$ - $h$ plane ${ }^{4}$. In the orthogonal $(n-2)$ subspace, the intersection space ${ }^{8}$, the degeneracy is retained at the first order ${ }^{10,30,32,33}$. The adiabatic energies of the two electronic states are indicated by $E_{1}$ and $E_{2}$, whereas $\mathbf{H}$ 
and $\mathbf{q}$ represent the electronic potential energy matrix and the nuclear coordinates, respectively.

In the direct algorithm proposed by Bearpark et al. ${ }^{15}$, the gradient used in the optimization is the following:

$$
\mathbf{g}=\mathbf{f}+\mathbf{g}_{p}
$$

Where:

$$
\mathbf{f}=2\left(E_{2}-E_{1}\right) \frac{\mathbf{x}_{1}}{\delta \kappa}=2 \Delta E \hat{\mathbf{x}}_{1}
$$

and

$$
\mathbf{g}_{p}=\mathbf{P} \frac{\partial E_{2}}{\partial \mathbf{q}}
$$

In Eq.(2), $\delta \kappa$ is the length of the gradient difference vector, i.e. $\delta \kappa=\sqrt{\mathbf{x}_{1}^{T} \mathbf{x}_{1}}$; and $\mathbf{P}$ represents the projector matrix which projects the gradient of the upper state $\left(\partial E_{2} / \partial \mathbf{q}\right)$ onto the subspace perpendicular to the plane $\left(\hat{\mathbf{x}}_{1}, \hat{\mathbf{x}}_{2}\right)$ :

$$
\mathbf{P}=\mathbf{1}-\hat{\mathbf{x}}_{1} \hat{\mathbf{x}}_{1}^{T}-\hat{\mathbf{x}}_{2} \hat{\mathbf{x}}_{2}^{T}
$$

The "hat" notation indicates a normalized vector. For what follows, it is worth noting that the projected gradient of the excited state energy is the same vector as that obtained by projecting the average of the two state gradients (for the proof see Appendix). This equivalence will be crucial in defining the second-derivative matrix of the seam energy.

The updated Hessian can become ill-conditioned in certain regions of the potential energy surface, if the composite gradient [Eq.(2)] is used throughout the optimization. 
When the energy difference is large, for instance, the Hessian is dominated by contributions arising from the branching plane ${ }^{17,34,35}$. As a result, the degeneracy region is rapidly reached. However, when the seam is located, contributions from the branching plane to the approximate Hessian may not vanish and therefore the degeneracy be lifted.

To improve the convergence of this algorithm we then propose to project both gradient and Hessian when the seam region is approached, so that the possible branching-space contamination is avoided. When the energy difference is below a certain threshold, we will combine an intersection-space displacement with a step taken along the gradient difference.

The potential energy within the intersection space can be can be described by a Taylor expansion truncated at the second-order:

$$
E_{\text {Seam }}=E_{\text {Seam }}^{0}+\left(\Delta \mathbf{q}_{I S}^{0}\right)^{T} \mathbf{g}_{I S}^{0}+\frac{1}{2}\left(\Delta \mathbf{q}_{I S}^{0}\right)^{T} \mathbf{H}_{I S}^{0} \Delta \mathbf{q}_{I S}^{0}
$$

Notice that $\Delta \mathbf{q}_{I S}^{0}=\mathbf{q}_{I S}-\mathbf{q}_{I S}^{0}$ is an $n$-dimensional vector. The zero-order term is taken as the reference point and set to zero. The seam energy gradient within the intersection space, $\mathbf{g}_{I S}$, is computed as the projected average gradient (see Appendix). This gradient allows us to introduce the intersection-space Hessian, recently proposed elsewhere ${ }^{9-11}$, as the second-derivatives matrix of the seam energy. Defining the intersection-space Hessian of the seam energy as the projected "derivative" of the seam energy gradient, we obtain: 


$$
\mathbf{W}_{I S}=\mathbf{P}\left[\frac{\partial}{\partial \mathbf{q}} \mathbf{g}_{I S}\right] \mathbf{P}=\frac{1}{2} \mathbf{P}\left[\frac{\partial}{\partial \mathbf{q}} \mathbf{P} \frac{\partial\left(E_{2}+E_{1}\right)}{\partial \mathbf{q}}\right] \mathbf{P}=\frac{1}{2} \mathbf{P}\left[\frac{\partial^{2}\left(E_{2}+E_{1}\right)}{\partial \mathbf{q}^{2}}-\frac{\lambda_{x_{1}}}{\delta \kappa} \frac{\partial^{2} \Delta E}{\partial \mathbf{q}^{2}}-\frac{\lambda_{x_{2}}}{\kappa_{12}} \frac{\partial^{2} H_{12}}{\partial \mathbf{q}^{2}}\right] \mathbf{P}
$$

In the differentiation we have used the definition of projection matrix reported in Eq.(2d). We have also introduced $\lambda_{x_{1(2)}}$ to indicate the components of the average gradient along the unit gradient difference (non adiabatic interstate coupling vector) and $\kappa_{12}$ for the length of the non-adiabatic interstate coupling vector.

To compute the Newton-Raphson displacement, the inverse of this Hessian matrix is required. However due to the projection, $\mathbf{W}_{I S}$ has two zero eigenvalues corresponding to the branching plane directions and therefore its inverse is not defined. However, using the idea of Peng et al. ${ }^{36}$, one can use instead the following matrix:

$$
\mathbf{H}_{I S}=\mathbf{W}_{I S}+(\mathbf{1}-\mathbf{P}) \mathbf{A}(\mathbf{1}-\mathbf{P})
$$

where $\mathbf{A}$ is diagonal matrix whose elements are set to a large constant (e.g. 1000) and $\mathbf{P}$ is the usual projection matrix defined in Eq.(2d). Consequently, using the Newton-Raphson method, the intersection-space displacement can be computed as:

$$
\Delta \mathbf{q}_{I S}^{0}=-\left(\mathbf{H}_{I S}^{0}\right)^{-1} \mathbf{g}_{I S}^{0}
$$

Although the displacement is an $m$-dimensional vector, the possible contributions from the branching plane have been projected out.

To guarantee the degeneracy, the following step along the gradient difference:

$$
\Delta \mathbf{q}_{B S}^{0}=\left(\Delta E_{0} / \delta \kappa_{0}\right) \hat{\mathbf{x}}_{1}^{0}
$$

is added to $\Delta \mathbf{q}_{I S}^{0}$, so that the total displacement is given by: 


$$
\Delta \mathbf{q}_{0}=\Delta \mathbf{q}_{I S}^{0}+\Delta \mathbf{q}_{B S}^{0}
$$

Although in principle the Hessian matrix, $\mathbf{W}_{I S}$, could be analytically computed ${ }^{9-11}$, an approximated Hessian is used in practise. Using the Broyden-Fletcher-Goldfarb-Shanno (BFGS) scheme, this matrix is calculated as:

$$
\mathbf{F}=\mathbf{F}^{0}+\frac{\Delta \mathbf{h}_{0}(\Delta \mathbf{h})_{0}^{T}}{\Delta \mathbf{h}_{0}^{T} \Delta \mathbf{q}_{0}}-\frac{\mathbf{F}^{0} \Delta \mathbf{q}_{0} \Delta \mathbf{q}_{0}^{T} \mathbf{F}^{0}}{\Delta \mathbf{q}_{0}^{T} \mathbf{F}^{0} \Delta \mathbf{q}_{0}}
$$

Here, $\Delta \mathbf{h}_{0}$ is computed as the difference between the projected average gradients [Eq.(2c)] evaluated at $\mathbf{q}$ (current iteration) and at $\mathbf{q}_{0}$ (previous iteration). $\Delta \mathbf{q}_{0}$ represents instead the difference between the current and previous geometry.

Table 1 - Relevant quantities used in the presented conical intersection optimization

\begin{tabular}{|c|c|c|}
\hline$\Delta E=E_{2}-E_{1}$ & $>$ Threshold $^{a}$ & $<$ Threshold \\
\hline Gradient $^{\mathrm{b}}$ & $\mathbf{g}=\mathbf{f}+\mathbf{g}_{I S}{ }^{\mathrm{c}}$ & $\mathbf{g}=\mathbf{g}_{I S}$ \\
\hline Total Displacement & $\Delta \mathbf{q}=-(\mathbf{F})^{-1} \mathbf{g}^{\mathrm{d}}$ & $\Delta \mathbf{q}=-\left(\mathbf{H}_{I S}\right)^{-1} \mathbf{g}_{I S}+(\Delta E / \delta \kappa) \hat{\mathbf{x}}_{1}{ }^{\mathrm{d}}$ \\
\hline \multicolumn{3}{|c|}{$\begin{array}{l}\text { The default cutoff has been set to } 5 \text { milliHartrees; }{ }^{\mathrm{b}} \text { this gradient is used to both update } \\
\text { the Hessian matrix and to compute the displacement; }{ }^{\mathrm{c}} \text { as defined in Eq. }(2) ;{ }^{\mathrm{d}} \mathbf{F} \text { is the } \\
\text { updated Hessian defined in Eq.(5) }\end{array}$} \\
\hline
\end{tabular}
algorithm.

In Table 1, we have reported the main features of the algorithm discussed in this paper. The reader may recognize some similarities between the proposed algorithm and the results obtained by Anglada et al. ${ }^{19,20}$, who have suggested applying the Han-Powell 
method ${ }^{17,18,34,37}$ to optimize conical intersection geometries. In proximity of the crossing seam, the two methodologies, for instance, use the same gradient (see Appendix) to update the Hessian matrix, $\mathbf{F}$ [Eq.(5)]. Moreover, the intersection-space Hessian $\mathbf{W}_{I S}$, used here, is similar to the reduced Hessian reported by Anglada et al.

Although the various similarities, the proposed algorithm differs from the one reported by Anglada et al. in several crucial points. Firstly, their method uses the Hessian only to compute the intersection space displacement throughout the optimization. Consequently, the method is known ( see for example Refs. ${ }^{17,20,34}$ ) to have a fairly slow convergence (or sometimes even no convergence at all) when one starts from a point too far away from the solution. In our algorithm, a composite gradient [Eq.(2)] is used to compute both the displacement and to update the Hessian in regions far away from the degeneracy. Some differences arise also in the proximity of the degeneracy region. In the seam region, the two proposed displacements differ in the form of the gradient used: we use the projected gradient [Eq.(2c)], whereas Anglada et al. propose using the reduced gradient. However, as theoretically outlined by Nocedal et al. ${ }^{34}$ and based on our own experience, the additional term present in reduced gradient does not provide any benefit to the overall convergence, once the crossing region is reached. Finally, the intersection-space Hessian, $\mathbf{W}_{I S}$, shown in Eq.(3b) is a symmetric $m$ by $m$ matrix, whereas the reduced Hessian used by Anglada et al. has dimension ( $m-2)$ by $(m-2)$. Nevertheless, it should be remarked that both matrices have the same $(m-2)$ rank, i.e. both matrices have $(m-2)$ non-zero eigenvalues. 
Along with minimum energy crossing points, the proposed algorithm is also capable to optimize different conical intersection structures along a constrained coordinate. We have recently proposed an implementation to carry out this type of optimization ${ }^{21}$, which prevents the possible occurrence of cancellation errors ${ }^{21-23}$. In that study, we suggested to apply first the constraints and subsequently to project out the branching space directions from the gradient. This procedure allowed us to map out successfully a region of $\mathrm{S}_{0} / \mathrm{S}_{1}$ fulvene crossing seam. Nevertheless in that study, we explicitly applied symmetry considerations to carried out the calculations. Here the procedure has been instead generalized and implemented in a development version of Gaussian software ${ }^{24}$. In Section 4, we will show an example of this type of computations.

Finally, we have explored the possibility of combining this new algorithm with the methodology implemented in Gaussian package to find transition state structures [see for example ${ }^{12}$ and references therein]. Routinely to compute a transition structure, one must compute the Hessian at a point located close enough to the quadratic region of the firstorder saddle point. This matrix must have one negative eigenvalue, and the corresponding eigenvector should be a suitable guess for the initial optimization direction. In this work, we follow the same procedure but limited to the intersection-space. Consequently, we begin computing the intersection-space Hessian ${ }^{9-11}$ at an initial conical intersection geometry. During the rest of the optimization, the intersection-space Hessian, $\mathbf{W}_{I S}$, is instead updated using the Bofill's scheme ${ }^{38}$ implemented in Gaussian, and then used as described above. In Section 4, we will report the results of a saddle point optimization onto the intersection hyperline of butadiene. 


\subsection{Computation of the minimum energy path within the intersection space.}

We now move to discuss the second algorithm proposed in this paper. This method enables one to compute a coordinate analogous to the intrinsic reaction coordinate (IRC), but confined to the intersection space (IS-IRC). Although several methods have been proposed to compute a segment of the intersection space [see for example ${ }^{7,20,22,28,39}$ and references therein], the algorithm that we have implemented is based upon the one proposed by Gonzalez et al. ${ }^{27}$. Thus, we will briefly present the ideas behind the original IRC algorithm; and we then will move to describe the few modifications required to compute directly the IS-IRC.

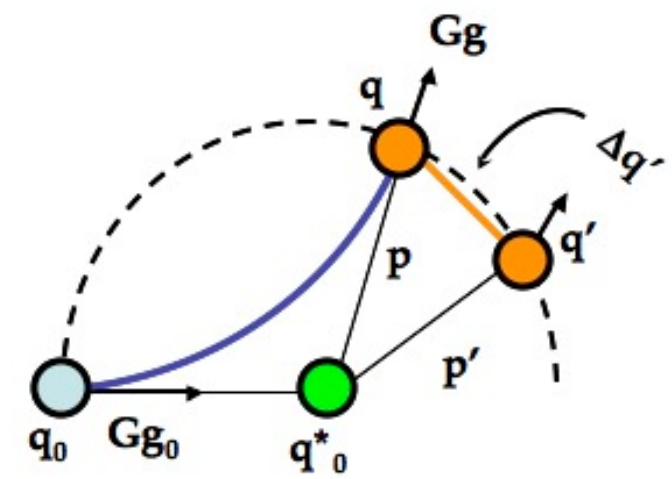

Figure 1 - A graphical representation of the vectors used in the original IRC algorithm ${ }^{27}$ is shown.

As shown in Figure 1, the original IRC algorithm ${ }^{26,27}$ was designed such that starting from a point $\mathbf{q}_{0}$, a $1 / 2 s$ displacement along the gradient $\mathbf{G g}_{0}$ is taken to locate $\mathbf{q}_{0}^{*}$ point, 
defined as the pivot point. From this pivot point, a constrained optimization is carried out on a surface of a hypershere of radius $1 / 2 s$ and centered at $\mathbf{q}_{0}^{*}$. Thus, in Figure $1, \mathbf{q}_{0}, \mathbf{q}^{\prime}$ and $\mathbf{q}$ are three points on the hypersphere and $\mathbf{q}_{0}^{*}$ the centre of it. Along the reaction path, at every $\mathbf{q}$ the residual gradient is, by construction, parallel to the $\mathbf{p}$ vector. Using this observation and the fact that the radius of the hypersphere must equal $1 / 2 s$, one obtains the following set of equations:

$$
\begin{gathered}
\Delta \mathbf{q}_{\mathrm{M}}=-\left(\mathbf{H}_{\mathrm{M}}-\sigma \mathbf{I}\right)^{-1}\left(\mathbf{g}_{\mathrm{M}}-\sigma \mathbf{p}_{\mathrm{M}}\right) \\
\Delta \mathbf{q}_{\mathrm{M}}^{T} \Delta \mathbf{q}_{\mathrm{M}}=\left[\mathbf{p}_{\mathrm{M}}-\left(\mathbf{H}_{\mathrm{M}}-\sigma \mathbf{I}\right)^{-1}\left(\mathbf{g}_{\mathrm{M}}-\sigma \mathbf{p}_{\mathrm{M}}\right)\right]^{T}\left[\mathbf{p}_{\mathrm{M}}-\left(\mathbf{H}_{\mathrm{M}}-\sigma \mathbf{I}\right)^{-1}\left(\mathbf{g}_{\mathrm{M}}-\sigma \mathbf{p}_{\mathrm{M}}\right)\right]=(1 / 2 s)^{2}
\end{gathered}
$$

Where the scalar $\sigma$ is the Lagrange multiplier, $\mathbf{I}$ is the identity matrix and the remaining quantities are defined in Table 2.

Table 2 - In the left column, the definition of the terms used in the original minimum energy path algorithm [Eq.(7)] are reported. On the right column are instead shown the definitions of the corresponding quantities used in the proposed algorithm. $\mathbf{G}$ is the Wilson matrix ${ }^{40} ; \mathbf{g}, \mathbf{H}$ and $\Delta \mathbf{q}$ indicate the gradient, the Hessian and the displacement, respectively .

Original Algorithm ${ }^{27}$

\section{Proposed Algorithm}

$$
\begin{array}{rlr}
\Delta \mathbf{q}_{\mathbf{M}} & =\mathbf{G}^{-1 / 2} \Delta \mathbf{q} & { }^{I S} \Delta \mathbf{q}_{\mathbf{M}}=\mathbf{G}^{-1 / 2} \Delta \mathbf{q}_{I S} \\
\mathbf{g}^{\prime}{ }_{\mathbf{M}}=\mathbf{G}^{1 / 2} \mathbf{g}^{\prime} & { }^{I S} \mathbf{g}_{\mathbf{M}}^{\prime}=\mathbf{P G}^{1 / 2} \mathbf{g}^{\prime} \\
\mathbf{H}_{\mathbf{M}}^{\prime}=\mathbf{G}^{1 / 2} \mathbf{H}^{\prime} \mathbf{G}^{1 / 2} & { }^{I S} \mathbf{H}_{\mathbf{M}}^{\prime}=\mathbf{P} \mathbf{G}^{1 / 2} \mathbf{W}_{I S}{ }^{\prime} \mathbf{G}^{1 / 2} \mathbf{P}+(\mathbf{1}-\mathbf{P}) \mathbf{A}(\mathbf{1}-\mathbf{P}) \\
\mathbf{p}_{\mathbf{M}}^{\prime}=\mathbf{G}^{-1 / 2} \mathbf{p}^{\prime} & { }^{I S} \mathbf{p}_{\mathbf{M}}^{\prime}=\mathbf{P G}^{-1 / 2} \mathbf{p}^{\prime}
\end{array}
$$


In order to compute the intersection space path, we follow the same derivation. However, all the quantities previously introduced are now projected onto the intersection space using the $\mathbf{P}$ matrix defined in Eq.(2d). Thus, replacing the various terms in Eq.(7) accordingly with Table 2, the intersection-space displacement, $\Delta \mathbf{q}_{I S}$, can be computed. However, due to the curved nature of the intersection space (see for instance Refs. ${ }^{1,9-11,33 \text {, }}$ ${ }^{41}$ ), this displacement will make the two crossing surfaces to split apart. To restore the degeneracy, an additional displacement within the branching space is finally added, in the spirit of the algorithm described above and in the way has been defined in Eq.(4c). As we will discuss in the next section, we have imposed convergence criteria on both gradient and displacement computed. Consequently, the degeneracy condition is implicitly required from Eq.(4c), since a large displacement would correspond to a large energy gap. 


\section{Computational Details}

The two algorithms described in the previous section have been implemented in a development version of Gaussian package ${ }^{24}$ and at two averaged states complete active space self consistent field level of theory (2-SA-CASSCF). Nevertheless, they can be easily implemented at any other level of theory, provided that analytical gradients are available $^{42}$. The overall convergence of the algorithm to optimize conical intersections has been tested on the $S_{0} / S_{1}$ crossing seam of benzene. The calculations have been carried out at the 2-SA-CASSCF level of theory, with a six $\pi$ electrons and six $\pi$ orbitals activespace and a STO-3G basis set. An active space of $(6 \pi, 6 \pi)$ and one of $(4 \pi, 4 \pi)$ were instead used to investigate the $S_{0} / S_{1}$ crossing seams of $z$-penta-3,5-diennium and 1,3butadiene, respectively. Also in these examples we have used the 2-SA-CASSCF level of theory, but with a $6-31 G^{*}$ basis set. In all the examples presented, the two crossing electronic states were equally weighted and the coupled perturbative MCSCF (CPMCSCF) equations solved to evaluate the gradients (see for instance ${ }^{43}$ ). However, tests have been also carried using approximated gradients, i.e. obtained without solving the CP-MCSCF equations, to guarantee the efficiency of the code implementation for molecules with either a big active space or with a large number of atoms. In these tests, the same structures (within $0.5 \mathrm{Kcal} / \mathrm{Mol}$ ) were optimized with and without computing the corrections for the gradients. Nevertheless, we have noticed that away from the seam region the two optimizations may differ significantly. Here, the displacement computed with the approximated gradient leads to higher energy geometries. Therefore, in general 
the two gradients may access the crossing seam in different areas and, consequently, lead to different optimized stationary points.

In the examples considered in the next section, a conical intersection geometry is considered converged when the largest component of the intersection-space gradient, or of ${ }^{I S} \mathbf{g}_{\mathbf{M}}^{\prime}$ (Table 1) for the second algorithm, is smaller than $4.5 \times 10^{-4}$ a.u. and with RMS below $3.0 \times 10^{-4}$ a.u. In addition to the gradient, also the maximum component of the total displacement is checked, $\Delta \mathbf{q}_{0}$ in Eq.(4c). A geometry is thus considered converged when the largest component of such a displacement is smaller than $1.8 \times 10^{-3}$ a.u. with RMS 1.2 x $10^{-3}$ a.u. 


\section{Results and Discussions}

The purpose of this section is to illustrate the potentialities of the two algorithms described in Section 2. We will begin comparing the performance of the proposed conical intersection algorithm using the $\mathrm{S}_{0} / \mathrm{S}_{1}$ and $\mathrm{S}_{0} / \mathrm{T}_{1}$ crossing seams of benzene. The z-penta-3,5-diennium crossing seam will be used to present an example of constrained optimization. The $S_{0} / S_{1}$ crossing seam of butadiene is examined with the aim of optimizing a saddle point onto this hyperline. We indeed optimized and characterized a new saddle point along this crossing seam. The potentialities and performance of the algorithm devised to compute the intersection-space path are finally showed on the $S_{0} / S_{1}$ crossing seam of butadiene.

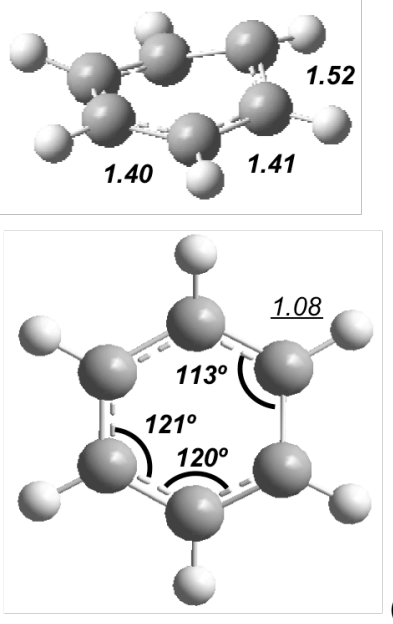

(a)

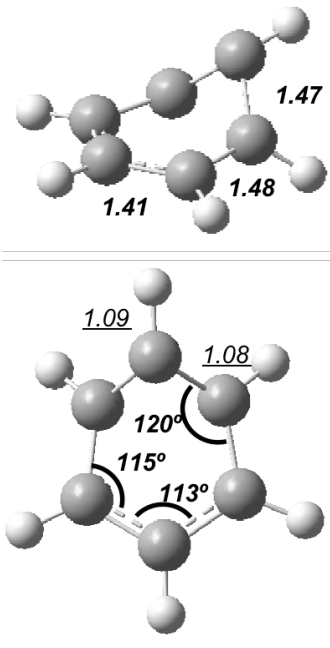

(b)

Figure 2 - Starting benzene geometry (a) and optimized $S_{0} / S_{1}$ conical intersection geometry (b) at SACASSCF(6,6)/STO-3G level. All the angles (italic) are reported in degrees, while the C-H (underlined) and $\mathrm{C}-\mathrm{C}$ bonds are reported in Angstrom.

4.1 Conical Intersection algorithm: To evaluate the overall efficiency of the conical intersection algorithm, the $S_{0} / S_{1}$ conical intersection seam of benzene (see for example Refs. ${ }^{44,45}$ ) has been used. We compare here the global convergence of the benzene $S_{0} / S_{1}$ conical intersection optimization computed 
by using the algorithm of Bearpark et al. ${ }^{15}$, the one proposed by Anglada et al. ${ }^{19}$ and the one proposed in this paper. All the conical intersection optimizations were started from a no-planar benzene structure obtained by distorting the minimum geometry (Figure $2 \mathrm{a}$ ) on the $\mathrm{S}_{1}$ potential energy surface ${ }^{44}$.

The same conical intersection geometry was optimized by the three different algorithms (Figure 2b), which is that previously reported by Bearpark et al. ${ }^{15}$. As noted in that study, a bigger basis set would, on one hand, provide a more accurate description of the molecular geometry. On the other hand, a bigger basis would not invalidate the results obtained regarding the convergence behavior. The objective of the test presented here is to discuss the total convergence of the new algorithm, so we used a relatively small basis set to speed up the tests.

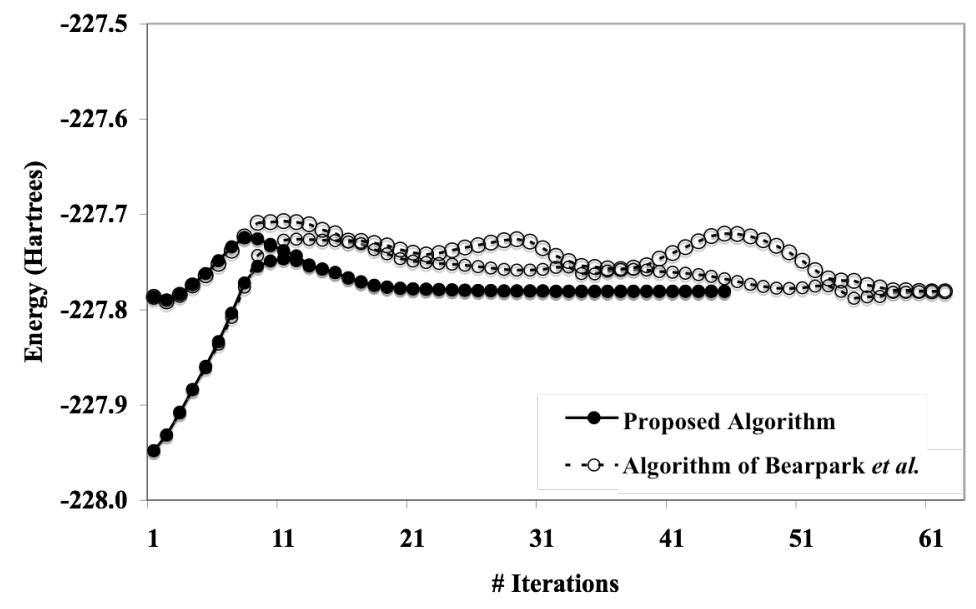

Figure 3 - Global convergence of an $S_{0} / S_{1}$ conical intersection optimization of benzene molecule: comparison between the energy profiles obtained by the algorithm of Bearpark et al. (open circles) and the proposed algorithm (filled circles). The energy changes of the two states during the optimization are reported.

In Figure 3 and Figure 4 we show the energies changes of the two $S_{0}$ and $S_{1}$ crossing states for 
benzene computed with the three algorithms during the optimization. As discussed in Section 2, the algorithm of Bearpark et al. (open circles in Figure 3) promptly reaches the crossing seam. However, once in that region, an ill-conditioned Hessian may slow the overall convergence. On the other hand, the algorithm proposed by Anglada et al. has a slower convergence towards the crossing seam (open circles in Figure 4) but it gives rise to a much smoother profile in the degeneracy region. In addition to the low rate of convergence to the degeneracy region, this latter algorithm tends to take steps in high-energy regions, where the molecule may, for example, dissociate and therefore be channeled towards high energetic portions of the intersection seam.

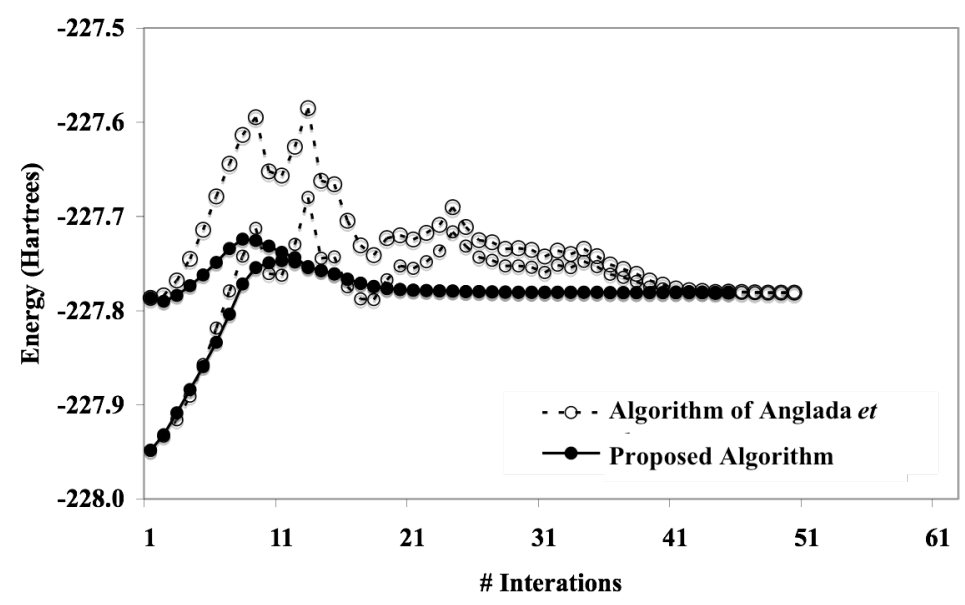

Figure 4 - Global convergence of an $\mathrm{S}_{0} / \mathrm{S}_{1}$ conical intersection optimization of benzene molecule: comparison between the method introduced by Anglada et al. (open circles) and the proposed algorithm (filled circles). The energy changes of the two states during the optimization are reported.

It is easily appreciated that the new algorithm (filled circles and solid line in both Figure 3 and Figure 4) has a faster convergence, when compared with both the other methods (open circles and dashed line in Figure 3 and Figure 4). In addition, it is clear that the implemented algorithm is capable to exploit the strength of the two methods in different regions of the optimization. It has the wanted convergence rate 
in the first part of the optimization, but it is also capable to maintained the degeneracy between the two crossing electronic states once the crossing seam is reached.

To summarize, we have noticed that the implemented algorithm is the fastest algorithm to converge (filled circles in Figure 3 and Figure 4) and is also capable to better retain the degeneracy between the two crossing states. To emphasize this last point, in Figure 5, the difference of the energies of the $S_{0}$ and $\mathrm{S}_{1}$ states of benzene is shown.

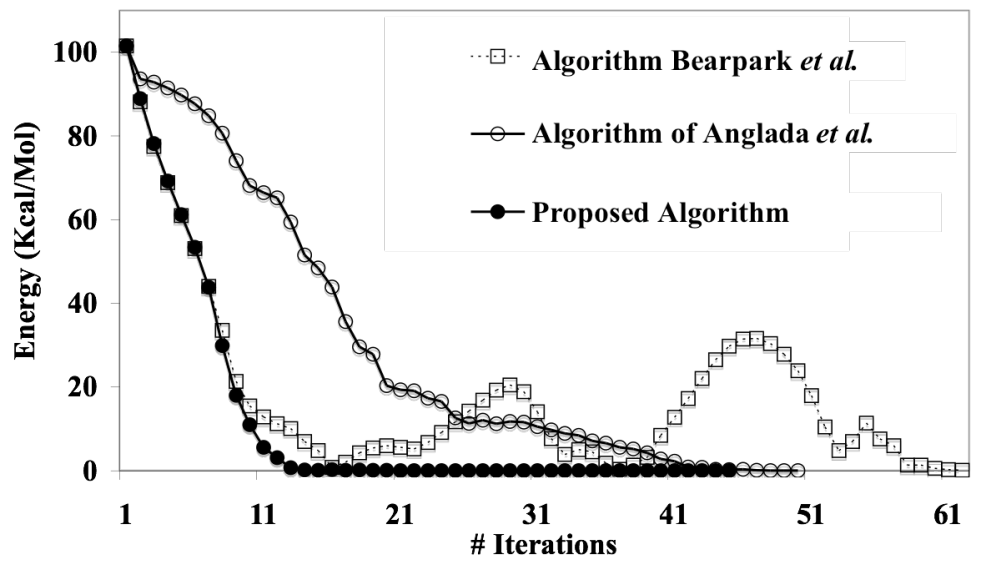

Figure 5 - Energy Difference (expressed in Kcal/Mol) between $S_{0}$ and $S_{1}$ electronic states in benzene during the conical intersection optimization. The results obtained with method of Anglada et al. (solid line and open circles) and the algorithm implemented by Bearpark et al.' (dash line and open squares) and the proposed algorithm (solid line and filled circles) are reported.

In Figure 5, it should be noted that the starting geometry is very poor, since the initial energy gap between the two states is of over $100 \mathrm{Kcal} / \mathrm{Mol}$. Such a difficult test demonstrates the robust global convergence of all the three algorithms. Nevertheless, the presented algorithm is the only one that keeps diminishing the energy gap between the two states constantly as the optimization proceeds. In addition, it shows fewer oscillations of the energy difference values. This result is consistent with having obtained 
a more accurate intersection-space Hessian with the update procedure.

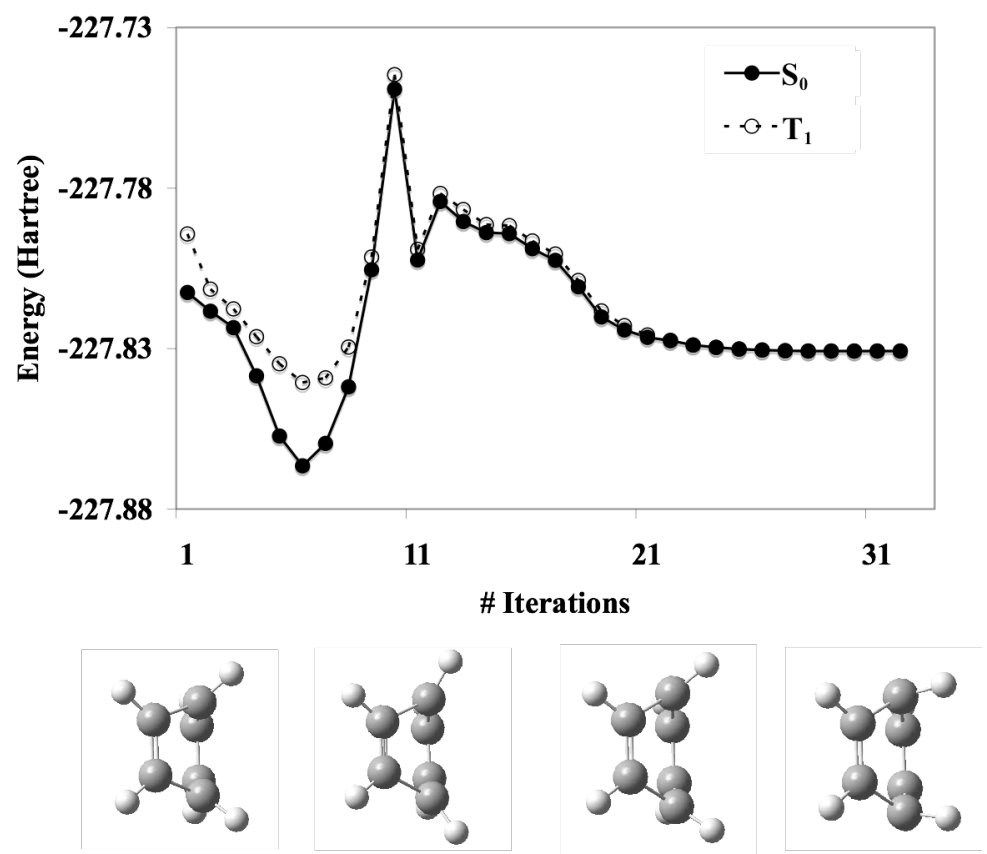

Figure 6 - Global convergence of an optimization of an $\mathrm{S}_{0} / \mathrm{T}_{1}$ crossing geometry of benzene. Geometries for selected points $(1,11,21,31)$ along the optimization path are shown at the bottom of the figure.

In Section 2, we have presented an algorithm to optimize conical intersections. However the same algorithm is also capable to optimize crossing points along (n-1)-dimensional crossing seams. In Figure 6, we show, as an example, the energy separation and the geometrical changes during the optimization of a crossing point along the $T_{1} / S_{0}$ crossing seam of benzene. As initial geometry (at iteration 1 in Figure 6), we have used boat-like structure, resembling the transition state connecting benzene to Dewar benzene ${ }^{44,46}$. Also in this type of crossing, the algorithm presented in this paper shows a robust overall convergence and it is capable to promptly approach the crossing seam. We note that the crossing point optimized in this test differs from the geometry obtained in our previous study ${ }^{15}$, as a consequence of choosing a different starting geometry. 


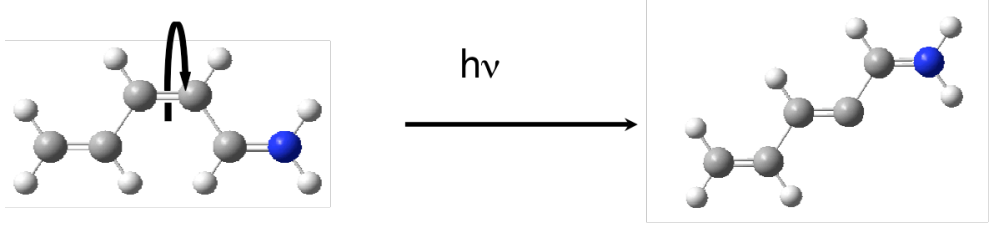

Scheme 1

To illustrate the generality of our implementation, we now show a conical intersection constrained optimization within the $\mathrm{S}_{0} / \mathrm{S}_{1}$ intersection space of $z$-penta-3,5-dieniminium cation ${ }^{22,47}$. We have carried out a relax scan of the central dihedral angle (Scheme 1) and in Figure 7a we report the energy profile computed. As previously reported $22,28,47,48$, we have found the minimum crossing point being in the region of $90^{\circ}$. However, it should be noted that the new algorithm is capable to achieve a high level of degeneracy (see for instance Refs. ${ }^{22,28}$ ) at all the optimized structures (Figure 7b).

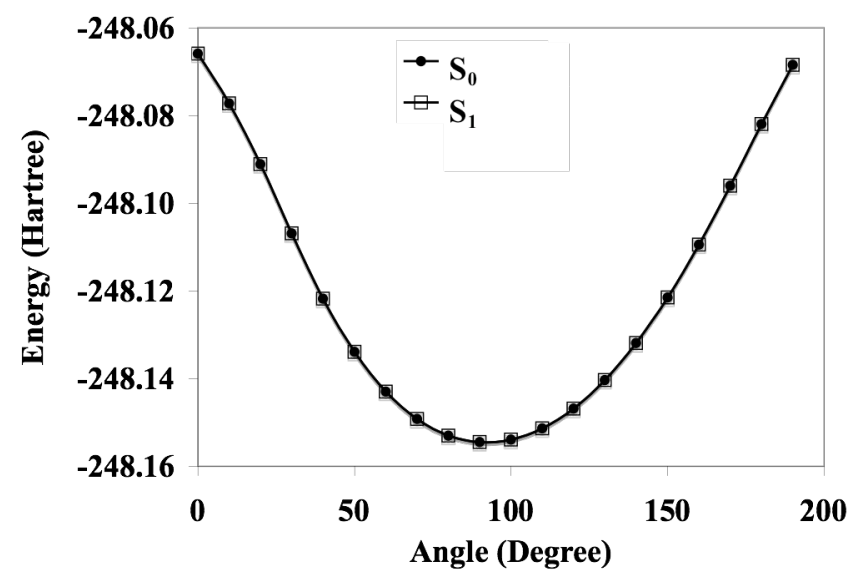

(a)

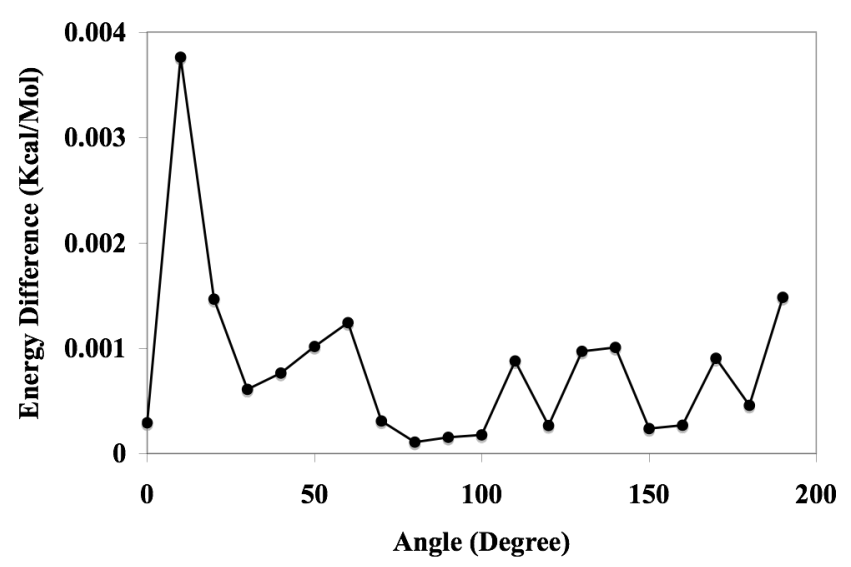

(b)

Figure 7 - Energy profile obtained as function of the z-penta-3,5-dieniminium cation central bond rotation (a). The degeneracy is reached for all the examined angles as showed in panel (b), where the energy difference (in $\mathrm{Kcal} / \mathrm{Mol}$ ) is reported. 
4.2 Algorithm to compute the intersection-space minimum energy path: To demonstrate the generality of the proposed conical intersection algorithm, we have optimized a saddle point along $\mathrm{S}_{0} / \mathrm{S}_{1}$ crossing seam of 1,3-butadiene. Such saddle point will then be linked up with two structures lower in energy along an intersection space minimum energy path computed with the second proposed algorithm.

In Section 2, we have mentioned that the presented algorithm can be combined with the algorithm implemented in Gaussian package to optimize transition structures. Generally, in order to optimize a saddle point structure, one must start from a geometry that is close enough to the quadratic region of such a point. In addition, the Hessian at this initial structure must show a negative eigenvalue, and the corresponding eigevector must be a suitable approximation for the direction connecting the two valleys. When one tries to optimize a saddle point within the intersection space, obviously, the initial geometry should also be a point of degeneracy.

In our first study ${ }^{49}$ on 1,3-budadiene, we had optimized three stationary point along the $\mathrm{S}_{0} / \mathrm{S}_{1}$ crossing seam: the cisoidal, the transoidal, and the central structure. In the present study, starting from that central geometry we first re-optimized the energy gap between the two states along the gradient difference vector. At this conical intersection the intersection-space Hessian was computed and an imaginary frequency (with corresponding eigenvector) obtained. Finally, using the new algorithm presented in this paper, we have been able to optimize a new saddle point never reported previously $\left({ }^{\mathbf{S P}} \mathbf{C I}_{\mathbf{8 2}}\right.$ in Figure 8). Such structure shows an imaginary frequency of some $639 i \mathrm{~cm}^{-1}$ corresponding to a combination of a $-\mathrm{CH}_{2}$ twisting mode and a symmetric rocking mode localized on $\mathrm{H}_{5}$ and $\mathrm{H}_{6}$. The seam normal mode corresponding to the imaginary frequency was then used to detect lower energy structures on the crossing seam, following a methodology recently proposed elsewhere ${ }^{11}$. Thus, we were able to locate one of the s-cisoidal conical intersection isomers $\left(\mathbf{C I}_{\mathbf{C i s}}\right.$ in Figure 8$)$ and another new conical 
intersection saddle point ( ${ }^{\mathrm{SP}} \mathbf{C I}_{66}$ in Figure 8).
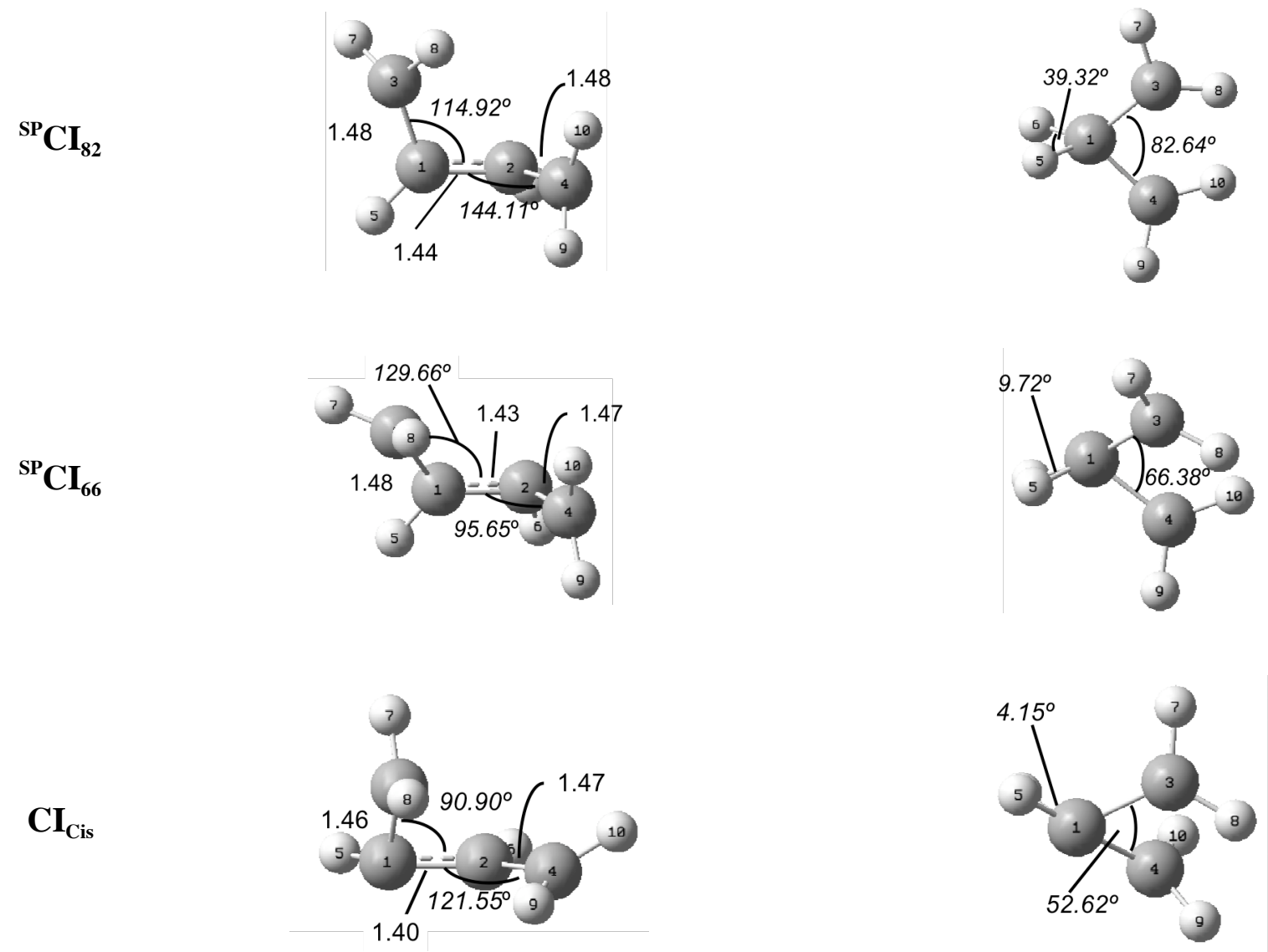

Figure 8 - Important geometric parameters for the optimized butadiene CI geometries found along the $\mathrm{S}_{0} / \mathrm{S}_{1} 1,3$-butadiene crossing seam. The bond lengths are expressed in angstroms, while the angles (in italic) are presented in degrees.

As pointed out elsewhere ${ }^{11}$, displacing along the mode with the imaginary frequency only suggest a possible connection between conical intersection structures, if lower energy structures exist. Nevertheless, a full reaction path computation restricted to the intersection space is required to infer rigorously that two conical intersection geometries are connected along the same seam. In Figure 9, we report the results obtained with the algorithm designed to compute the minimum energy path within the 


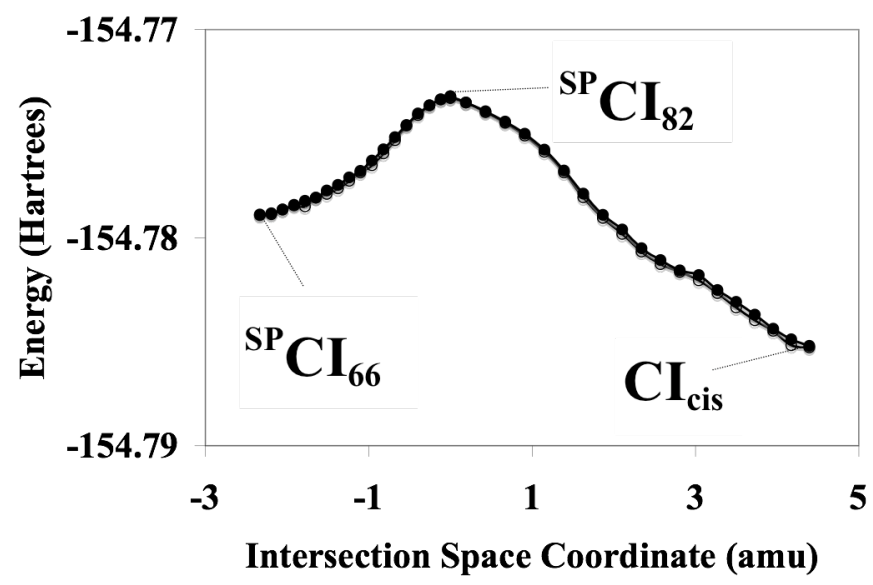

(a)

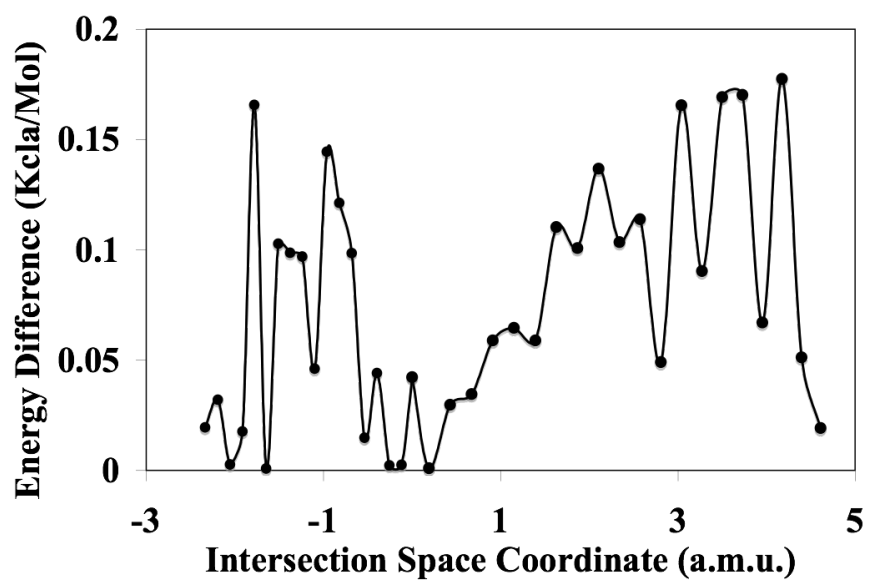

(b)

Figure 9 - Minimum energy path connecting ${ }^{\mathrm{SP}} \mathbf{C I}_{\mathbf{6 6}}$ (negative part of the intersection space coordinate in

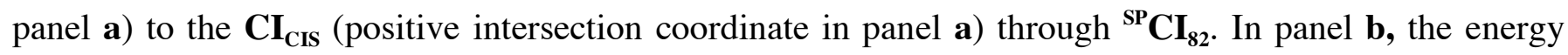
difference computed at each point along the intersection space minimum energy coordinate is reported.

The accuracy of the algorithm proposed in this paper can be appreciated from Figure 9b; where the energy difference at each optimized structure along the path is reported. It can be seen that the energy gap is well below the chemical accuracy $(1 \mathrm{Kcal} / \mathrm{Mol})$ and all the structure computed can be indeed be thought as points belonging to the same portion of the $S_{0} / S_{1} 1,3$-btadiene crossing seam.

We have started our computations from the structure ${ }^{\text {SP }} \mathbf{C I}_{\mathbf{8 2}}$ (Figure 8) and we have used as initial relaxation direction (see for instance Refs ${ }^{12,26,27,50}$ ) the eigenvector corresponding to the imaginary frequency. It should be emphasized that the absence of discontinuity in such profile is the evidence of a single crossing seam. Thus, we can conclude that the ${ }^{\mathrm{SP}} \mathbf{C I}_{\mathbf{8 2}}$ is the highest in energy structure along the 
portion of intersection space connecting $\mathbf{C I}_{\mathbf{C i s}}$ and ${ }^{\mathbf{S P}} \mathbf{C I}_{\mathbf{6 6}}$. We conclude noticing that due to the complexity morphology of area around the ${ }^{\mathbf{S P}} \mathbf{C I}_{6 \mathbf{6}}$, in our calculations the step length was reduced. This explains the number of points on the left hand side of the profile in Figure 9a. 


\section{Conclusions}

In this paper we have presented two new algorithms to study the extended nature of the crossing seam. The first algorithm represents an improvement over the already available algorithms. It combines an existing fast convergence in reaching the seam region with an extreme flexibility in the degeneracy region itself. We have demonstrated the potentialities of this algorithm on different crossing seams and with different scopes. We have optimized both minima and saddle points on crossing seams as well as carried out conical intersection optimization along a frozen internal coordinate. In all cases, the results obtained show a fast and smooth convergence to an optimized conical intersection.

The second algorithm has instead been designed to compute the intersection-space minimum energy path. Adapting one of the available algorithms ${ }^{27}$ to compute the reaction path on the entire potential energy surface, we have implemented an analogous method limited exclusively to the intersection space. We have shown the potentialities by explicitly mapping out a finite region of the $\mathrm{S}_{0} / \mathrm{S}_{1} 1,3$ butadiene crossing seam.

\section{Acknowledgment}

L.B. is financed by the Spanish/Ministerio de Educación y Ciencia/ for Project No. CTQ2005-04563 and the Ramón y Cajal program. F.S. is pleased to acknowledge the support of Gaussian, Inc. and COST for sponsoring his visit to the University of Girona. 


\section{Appendix}

In this Appendix, we will show the equivalence between the gradient of one of the two crossing states energy and the average gradients, once they are both projected onto the first-order intersection space. Using the definition of projection matrix given in Eq.(2c), the projected gradient, for instance, of the state 2 can be written as:

$$
\mathbf{P} \frac{\partial E_{2}}{\partial \mathbf{q}}
$$

On the other hand the projected average gradient is defined as:

$$
\frac{1}{2} \mathbf{P} \frac{\partial\left(E_{2}+E_{1}\right)}{\partial \mathbf{q}}
$$

Proving their difference zero is equivalent to show that they are the same vector. Thus:

$$
\frac{1}{2} \mathbf{P} \frac{\partial\left(E_{2}+E_{1}\right)}{\partial \mathbf{q}}-\mathbf{P} \frac{\partial E_{2}}{\partial \mathbf{q}}=\frac{1}{2} \mathbf{P} \frac{\partial\left(E_{1}-E_{2}\right)}{\partial \mathbf{q}}=\frac{1}{2} \mathbf{P} \mathbf{x}_{1}=\frac{1}{2}\left(\mathbf{x}_{1}-\mathbf{x}_{1}\right)=\mathbf{0}
$$

In Eq.(A.3), we have used the definition of the projection matrix $\mathbf{P}$ [Eq.(2)] and exploited the fact that the gradient difference vector and the non-adiabatic intrastate coupling vectors are chosen to be orthonormal to each other. 


\section{Reference}

(1) Domcke, W.; Yarkony, D. R.; Koppel, H., Conical Intersections: Electronic Structure, Dynamics \& Spectroscopy. ed.; World Scientific Publishing Co. : Singapore, 2004; Vol. 15.

(2) Bernardi, F.; Olivucci, M.; Robb, M. A., Chem. Soc. Rev. 1996, 25, 321.

(3) Kessinger, M.; Michl, J., Excited States and Photochemistry of Organic Molecules. WileyVCH: New York, 1995.

(4) Yarkony, D. R., Rev. Mod. Phys. 1996, 68, 985.

(5) Toniolo, A.; Levine, B.; Thompson, A.; Quenneville, J.; Ben-Nun, M.; Owens, J.; Olsen, S.; Manohar, L.; Martinez, T. J., Photochemistry from First Principles and Direct Dynamics in Computational Methods. In Organic Photochemistry, Kutateladze, A., Marcel-Dekker: New York, 2005.

(6) Ben-Nun, M.; Martinez, T. J., Chem. Phys. Lett. 1998, 298, 57; Boggio-Pasqua, M.; Bearpark, M. J.; Hunt, P. A.; Robb, M. A., J. Am. Chem. Soc. 2002, 124, 1456; Boggio-Pasqua, M.; Bearpark, M. J.; Ogliaro, F.; Robb, M. A., J. Am. Chem. Soc. 2006, 128, 10533; Boggio-Pasqua, M.; Ravaglia, M.; Bearpark, M. J.; Garavelli, M.; Robb, M. A., J. Phys. Chem. A 2003, 107, 11139.

(7) Laino, T.; Passerone, D., Chem. Phys. Lett. 2004, 389, 1.

(8) Atchity, G. J.; Xantheas, S. S.; Ruedenberg, K., J. Chem. Phys. 1991, 95, 1862.

(9) Paterson, M. J.; Bearpark, M. J.; Robb, M. A.; Blancafort, L., J. Chem. Phys. 2004, 121, 
(10) Sicilia, F.; Blancafort, L.; Bearpark, M. J.; Robb, M. A., J. Phys. Chem. A 2007, 111, 2182

(11) Sicilia, F.; Blancafort, L.; Bearpark, M. J.; Robb, M. A., Theo. Chem. Acc. 2007, 118, 241.

(12) Schlegel, H. B., J. Comp. Chem. 2002, 24, 1514.

(13) Yarkony, D. R., J. Chem. Phys. 1990, 92, 2457.

(14) Dallos, M.; Lischka, H.; Shepard, R.; Yarkony, D. R.; Szalay, P. G., J. Chem. Phys. 2004, 120, 7330; Ragazos, I. N.; Robb, M. A.; Bernardi, F.; Olivucci, M., Chem. Phys. Lett. 1992, 197, 217.

(15) Bearpark, M. J.; Robb, M. A.; Schlegel, H. B., Chem. Phys. Lett. 1994, 223, 269.

(16) Chachiyo, T.; Rodriguez, J. H., J. Chem. Phys. 2005, 123, 094711; Yamazaki, S.; Kato, S., J. Chem. Phys. 123, 123, 114510; Izzo, R.; Klessinger, M., J. Comp. Chem. 2000, 21, 52; Page, C. S.; Olivucci, M., J. Comp. Chem. 2003, 24, 298.

(17) Fletcher, R., Practical Methods of Optimization. Wiley \& Sons: New York, 1981; Vol. 2.

(18) Gill, P. E.; Murray, W., Numerical Methods for Constrained Optimization. Academic Press: London, 1974.

(19) Anglada, J. M.; Bofill, J. M., J. Comp. Chem. 1996, 18, 992.

(20) De Vico, L.; Olivucci, M.; Lindh, R., J. Chem. Theor. Comp. 2005, 1, 1029.

(21) Bearpark, M. J.; Blancafort, L.; Paterson, M. J., Mol. Phys. 2006, 104, 1033. 
(22) Migani, A.; Robb, M. A.; Olivucci, M., J. Am. Chem. Soc. 2003, 125, 2804.

(23) Takahashi, O.; Sumita, M., J. Chem. Phys. 2004, 121, 7030.

(24) Frisch, M. J.; Trucks, G. W.; Schlegel, H. B.; Scuseria, G. E.; Robb, M. A.; Cheeseman, J. R.; Montgomery, J. A., Jr.; Vreven, T.; Scalmani, G.; Kudin, K. N.; Iyengar, S. S.; Tomasi, J.; Barone, V.; Mennucci, B.; Cossi, M.; Rega, N.; Petersson, G. A.; Nakatsuji, H.; Hada, M.; Ehara, M.; Toyota, K.; Fukuda, R.; Hasegawa, J.; Ishida, M.; Nakajima, T.; Honda, Y.; Kitao, O.; Nakai, H.; Li, X.; Hratchian, H. P.; Peralta, J. E.; Izmaylov, A. F.; Heyd, J. J.; Brothers, E.; Staroverov, V.; Zheng, G.; Kobayashi, R.; Normand, J.; Burant, J. C.; Millam, J. M.; Klene, M.; Knox, J. E.; Cross, J. B.; Bakken, V.; Adamo, C.; Jaramillo, J.; Gomperts, R.; Stratmann, R. E.; Yazyev, O.; Austin, A. J.; Cammi, R.; Pomelli, C.; Ochterski, J. W.; Ayala, P. Y.; Morokuma, K.; Voth, G. A.; Salvador, P.; Dannenberg, J. J.; Zakrzewski, V. G.; Dapprich, S.; Daniels, A. D.; Strain, M. C.; Farkas, O.; Malick, D. K.; Rabuck, A. D.; Raghavachari, K.; Foresman, J. B.; Ortiz, J. V.; Cui, Q.; Baboul, A. G.; Clifford, S.; Cioslowski, J.; Stefanov, B. B.; Liu, G.; Liashenko, A.; Piskorz, P.; Komaromi, I.; Martin, R. L.; Fox, D. J.; Keith, T.; Al-Laham, M. A.; Peng, C. Y.; Nanayakkara, A.; Challacombe, M.; Chen, W.; Wong, M. W.; Pople, J. A. Gaussian DV, Revision F.01, Gaussian, Inc.: Wallingford, CT, 2006.

(25) Fukui, K., Acc. Chem. Res. 1981, 14, 363.

(26) Gonzalez, C.; Schlegel, H. B., J. Chem. Phys. 1989, 90, 2154.

(27) Gonzalez, C.; Schlegel, H. B., J. Chem. Phys. 1990, 94, 5523.

(28) Migani, A.; Sinicropi, A.; Ferre, N.; Cembran, A.; Garavelli, M.; Olivucci, M., Faraday Discuss. 2004, 127, 179.

(29) We can regard the intersections occurring along a (n-1)-dimensional hyperline, e.g. between 
two states of different spin multiplicity, as a subgroup of this more general class of electronic states intersections and therefore they will not be discussed any further.

(30) Yarkony, D. R., J. Chem. Phys. 2005, 123, 204101.

(31) Redmon, L. T., Phys. Rev. A 1982, 25, 2453.

(32) Mead, C. A., J. Chem. Phys. 1983, 78, 807; Young, R. A.; Yarkony, D. R., J. Chem. Phys. 2006, 125, 234301.

(33) Yarkony, D. R., J. Chem. Phys. 2005, 123, 134106.

(34) Nocedal, J.; Overton, M. L., SIAM J. Num. Analysis 1985, 22, 821.

(35) Toniolo, A.; Ben-Nun, M.; Martinez, T. J., J. Phys. Chem. A 2002, 106, 4679.

(36) Peng, C.; Ayala, P. Y.; Schlegel, H. B.; J., F. M., J. Comp. Chem. 1996, 17, 49.

(37) Wright, S. J., Algorithms and Software for Linear and Nonlinear Programming. In Foundations of Computer-Aided Process Design, CACHE Publications: 1999.

(38) Bofill, J. M., J. Comp. Chem. 1994, 15, 1.

(39) Passerone, D.; Laino, T., Comp. Phys. Comm. 2005, 169, 305.

(40) Wilson, E. B.; Decius, G. C.; Cross, P. C., Molecular Vibrations. McGraw-Hill: London, 1955.

(41) Yarkony, D. R., J. Chem. Phys. 2005, 123, 1234106. 
(42) In the case of a proper conical intersection the non-adiabatic interstate coupling vector is also required.

(43) Almlöf, J.; Taylor, P., Int. J. Quantum Chem. 1985, 27, 743.

(44) Palmer, I. J.; Ragazos, I. N.; Bernardi, F.; Olivucci, M.; Robb, M. A., J. Am. Chem. Soc. 1993, $115,673$.

(45) Domcke, W.; Sobolewski, A. L.; Woywod, C., Chem. Phys. Lett. 1993, 203, 220; Sobolewski, A. L.; Woywod, C.; Domcke, W., J. Chem. Phys. 1993, 98, 5627.

(46) Turro, N. J., Modern Molecular Photochemistry. Benjamin Cummings: Menlo Park, 1978.

(47) Garavelli, M.; Celani, P.; Bernardi, F.; Robb, M. A.; Olivucci, M., J. Am. Chem. Soc. 1997, 119; Weingart, O.; Migani, A.; Olivucci, M.; Robb, M. A.; Buss, V.; Hunt, P., J. Phys. Chem. A 2004, 108, 4685; Vreven, T.; Bernardi, F.; Garavelli, M.; Olivucci, M.; Robb, M. A.; Schlegel, H. B., J. Am. Chem. Soc. 1997, 119, 12687.

(48) Garavelli, M.; Bernardi, F.; Olivucci, M.; Vreven, T.; Klein, S.; Celani, P.; Robb, M. A., Faraday Discuss. 1998, 110, 51.

(49) Olivucci, M.; Ragazos, I. N.; Bernardi, F.; Robb, M. A., J. Am. Chem. Soc. 1993, 115, 3710.

(50) Celani, P.; Robb, M. A.; Garavelli, M.; Bernardi, F.; Olivucci, M., Chem. Phys. Lett. 1995, 243,1 . 